\title{
mesure des contraintes in situ par la cellule de surcarottage CERCHAR
}

par

H. M. Helal

\section{Introduction}

Depuis un certain nombre d'années, le problème de la mesure des contraintes in situ préoccupe bon nombre de chercheurs en mécanique des terrains. L'intérêt que présenterait une bonne connaissance de l'état de contrainte des massifs rocheux n'est plus à démontrer, et cette recherche a fait l'objet de diverses contributions : Leeman (1), Bonnechère (2)*, etc. Des symposiums, comme Zurich en 1977, lui ont même été entièrement consacrés.

Certains chercheurs ont orienté leurs travaux vers des tentatives de modélisation théorique des sites étudiés: d'autres ont préféré la mise au point de techniques et d'instruments permettant d'effectuer des mesures en place. Le tableau I ci-après rassemble quelques exemples de ces dispositifs et rappelle les principes de leur conception.

Le CERCHAR pour sa part, et dans le cadre de ses études relatives à la stabilité des exploitations à faible profondeur, a été amené à mesurer couramment les contraintes in situ, et à comparer systématiquement les valeurs obtenues aux résultats issus de calculs sur modèles. Deux techniques sont habituellement utilisées - Bonvallet ( 3 ) - :

- la méthode de la rosette, basée sur la mesure des déformations,

- la méthode du vérin plat, basée sur l'équilibrage des pressions.

L'intérêt de ces deux méthodes, relativement faciles à mettre en œuvre, souffre du fait que les mesures ne peuvent être faites qu'à la paroi des ouvrages. Or dans cette zone la roche est souvent dans un état plastique ou bien soumise à une importante fracturation d'origine mécanique.

* Les chiffres entre parenthèses renvoient à la bibliographie placée en fin d'article.
Dans ces conditions, seules les mesures réalisées à partir d'un forage peuvent atteindre une profondeur suffisante pour pouvoir s'affranchir de ce handicap. Le CERCHAR a donc cherché à se doter d'une technique et d'un matériel lui permettant d'utiliser la technique du surcarottage, technique particulièrement bien adaptée à la mesure des contraintes dans le cœur des piliers ou dans le toit d'une exploitation.

Le principe de la méthode consiste à détendre la roche autour d'un sondage en mesurant les déformations diamétrales provoquées par cette opération. La mesure précise de ces déformations permet de remonter aux contraintes, moyennant la connaissance des caractéristiques élastiques $(E, v)$ du matériau.

A cet effet, une cellule dilatométrique à mesure diamétrales a été conçue et mise au point, ainsi qu'un ensemble de matériels destiné à déterminer en outre un module pressiométrique du matériau.

Le texte qui suit décrit la cellule CERCHÁR, son principe, son mode d'utilisation, le matériel de mesure qui lui est attaché, ainsi que les bases théoriques sur lesquelles les résultats obtenus sont interprétés. Quelques exemples d'utilisation de cette cellule en laboratoire et au chantier sont également présentés.

\section{La procédure de surcarottage}

Nous ne décrirons pas ici le principe du surcarottage, déjà publié par ailleurs (Leeman (1), Bonnechère (2), etc.). Nous rappellerons simplement la procédure adoptée par le CERCHAR.

Dans une première étape, on fore un trou de diamètre assez grand $(210 \mathrm{~mm})$ jusqu'à la profondeur à laquelle on veut mesurer les contraintes (fig. 1a). On réalise ensuite un second forage concentrique au premier mais de diamètre plus petit $(97 \mathrm{~mm}$ ) (fig. 1 b). La cellule est alors introduite dans ce petit forage, et les conditions initiales sont mesurées et enregistrées 

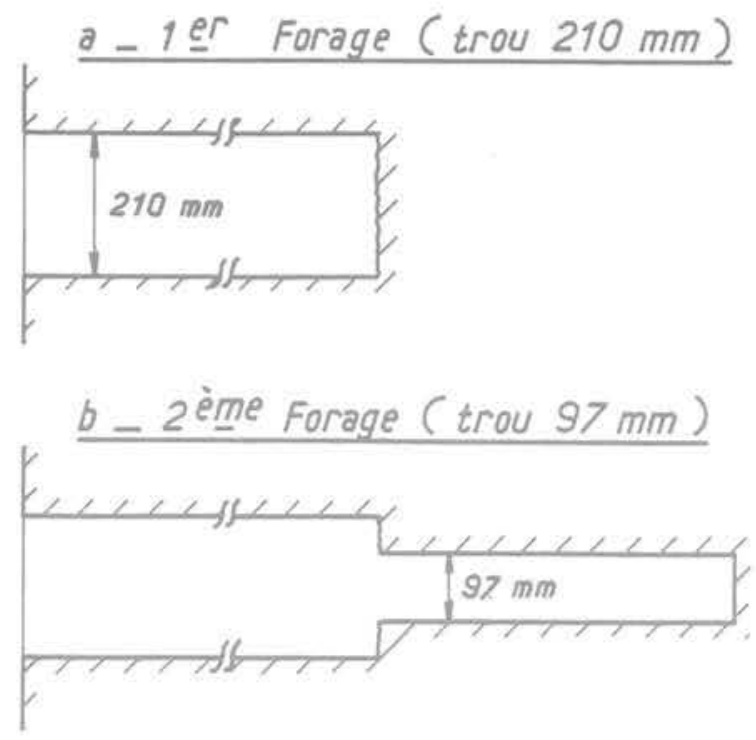

c - Installation de la cellule
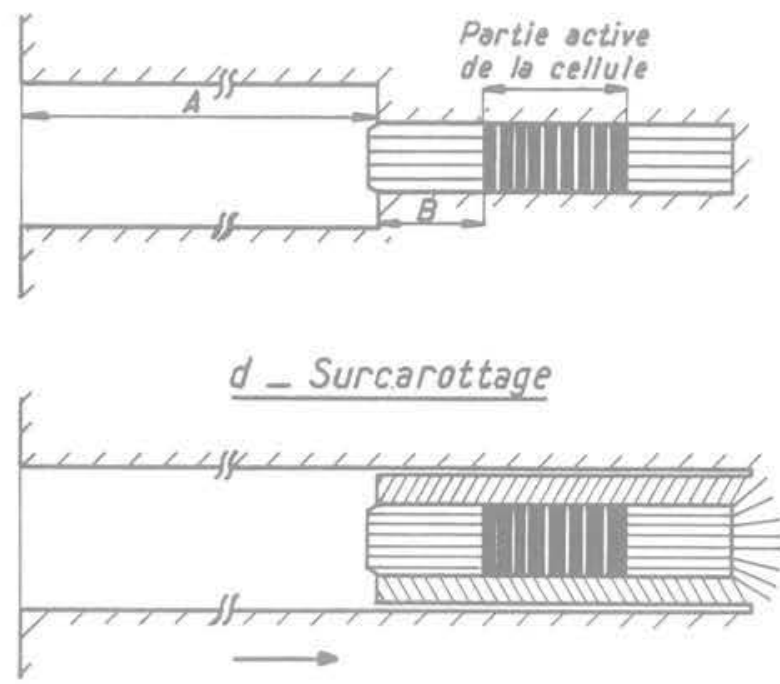

Fig. 1 La procédure du surcarottage (diamètre initial du trou) (fig. 1c). Trois cycles pressiométriques de mise en pression et de décharge sont effectués, en vue de déterminer le module d'élasticité du massif. Une fois ces cycles terminés, le surcarottage proprement dit est exécuté; les terrains autour de la cellule de mesure sont surcarottés en grand diamètre $(210 \mathrm{~mm})$ pendant que l'on mesure simultanément les variations de diamètre selon trois directions (fig. 1d).

\section{La cellule CERCHAR}

La cellule CERCHAR est une cellule dilatométrique fournissant des mesures diamétrales avec une résolution inférieure au micron, et qui peut fonctionner sous très faible pression.

Le prototype de l'appareil a été construit en coopération avec les sociétés Mazier et $\mathrm{C}^{\mathrm{ie}}$ et Coyne et Bellier. II est conçu pour être utilisé dans un trou de forage de diamètre $97 \mathrm{~mm}$. Un schéma de la cellule est donné par la figure 2, et une photo de l'appareil dans son montage d'étalonnage est présentée à la figure 3 .

La cellule se présente sous la forme d'un cylindre de $500 \mathrm{~mm}$ de longueur et de $95 \mathrm{~mm}$ de diamètre. Le corps en acier est enveloppé par une manchette de caoutchouc souple. L'étanchéité est assurée, à chacune des deux extrémités par un manchon tronconique qui assure le blocage de la manchette sur le corps métallique.

Six capteurs de déplacement sont logés dans la partie centrale de la cellule dont la partie gonflable mesure $200 \mathrm{~mm}$. Ils sont disposés en trois paires décalées de 120 degrés les unes par rapport aux autres, et sont placés dans trois plans distants de $40 \mathrm{~mm}$. Pour tenir compte d'une éventuelle dérive des capteurs due, par exemple, à une variation de la température en cours d'essais, un septième capteur bloqué est installé dans le corps de la cellule et sert de référence.

Les capteurs sont de fabrication Coyne et Bellier. Chacun est constitué d'une bobine d'induction montée sur un anneau de quartz et d'un disque de laiton mobile par rapport à ce bobinage. Le circuit électronique contient un oscillateur et un amplificateur alimentés par une tension stabilisée. La grandeur caractéristique de la mesure du déplacement du disque de laiton par rapport à la bobine d'induction est la fréquence du courant alternatif parcourant le circuit.

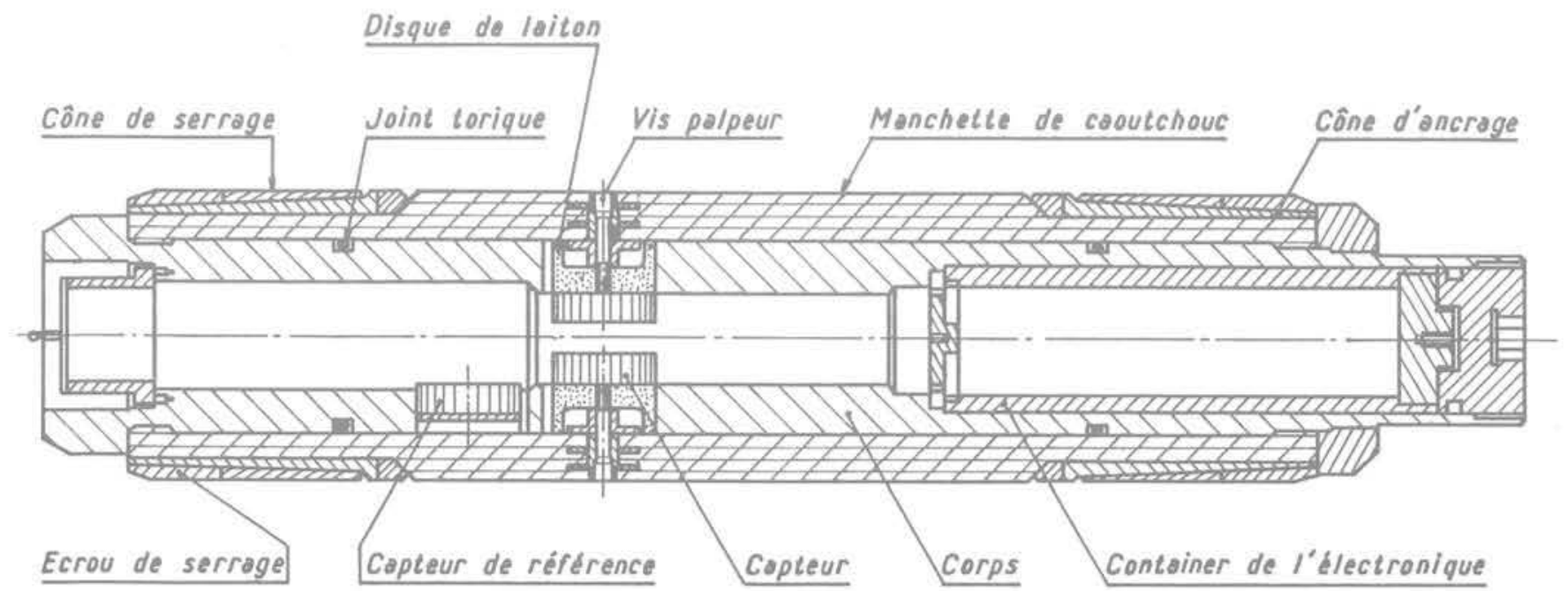

Fig. 2 Plan schématique de la cellule CERCHAR 


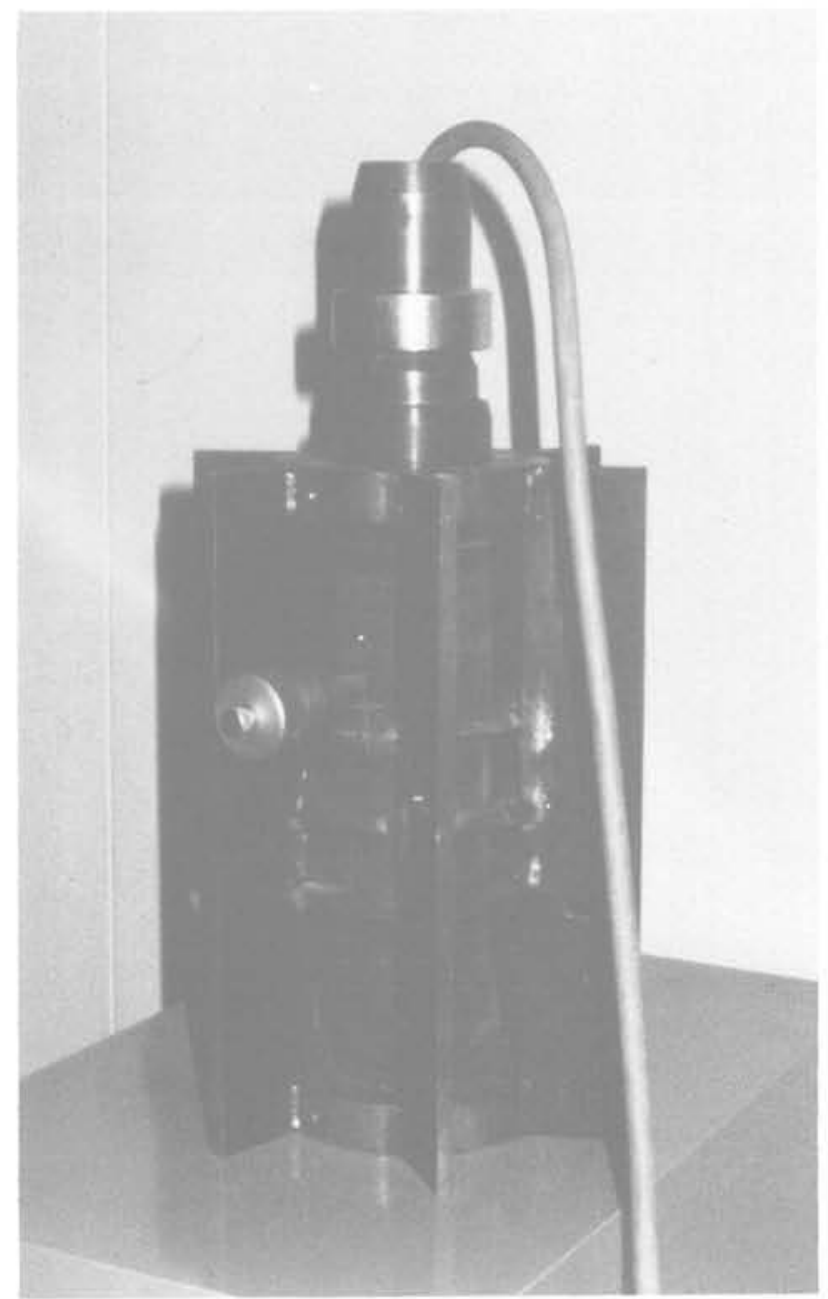

Fig. 3 La cellule CERCHAR dans son montage d'étalonnage

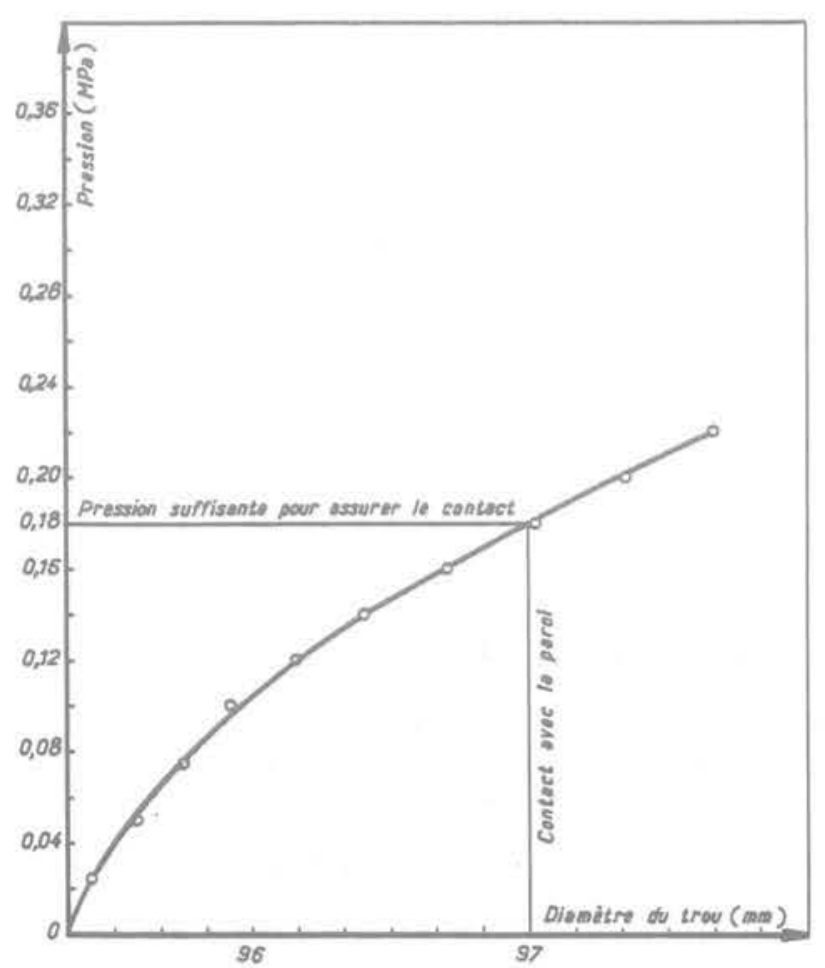

Fig. 4 Courbe caractéristique du gonflement de la manchette $n^{\circ} 3$
Le disque de chaque capteur est fixé dans la manchette par une vis palpeur munie d'un joint torique pour assurer l'étanchéité de la cellule.

Pour assurer le contact entre la cellule et la paroi du forage, la cellule est gonflée par de l'azote sous pression. La pression nécessaire pour assurer ce contact est une fonction de deux paramètres principaux: la souplesse de la manchette utilisée, dont dépend la pression maximale que I'on peut appliquer lors de l'essai pressiométrique, et la résistance à la traction du matériau dans lequel l'essai est réalisé, pour éviter de casser l'anneau du surcarottage pendant l'opération. La figure 4 montre un exemple de courbe de gonflement de la manchette utilisée dans la craie. Dans ce cas, la pression suffisante pour assurer le contact des six capteurs avec la paroi est de l'ordre de $0,18 \mathrm{MPa}$. La pression retenue pour opérer dans la craie est de 0,2 $\mathrm{MPa}$, pour assurer le contact même en cas d'irrégularité des parois du forage.

Afin de maintenir cette pression constante pendant toute l'opération, un système de détendeur asservi est utilisé. II permet un double passage de l'azote, c'est-à-dire de la bouteille vers la cellule, mais également de la cellule vers l'extérieur.

\section{4 Étalonnage des capteurs}

La courbe de réponse de chaque capteur c'est-à-dire l'évolution de la fréquence du courant dans le circuit oscillant en fonction du déplacement du disque - est obtenue par l'étalonnage des capteurs. Cet étalonnage se fait à l'aide du montage présenté figure 3 qui permet de fixer la cellule et une vis micrométrique dont la précision est de deux microns.

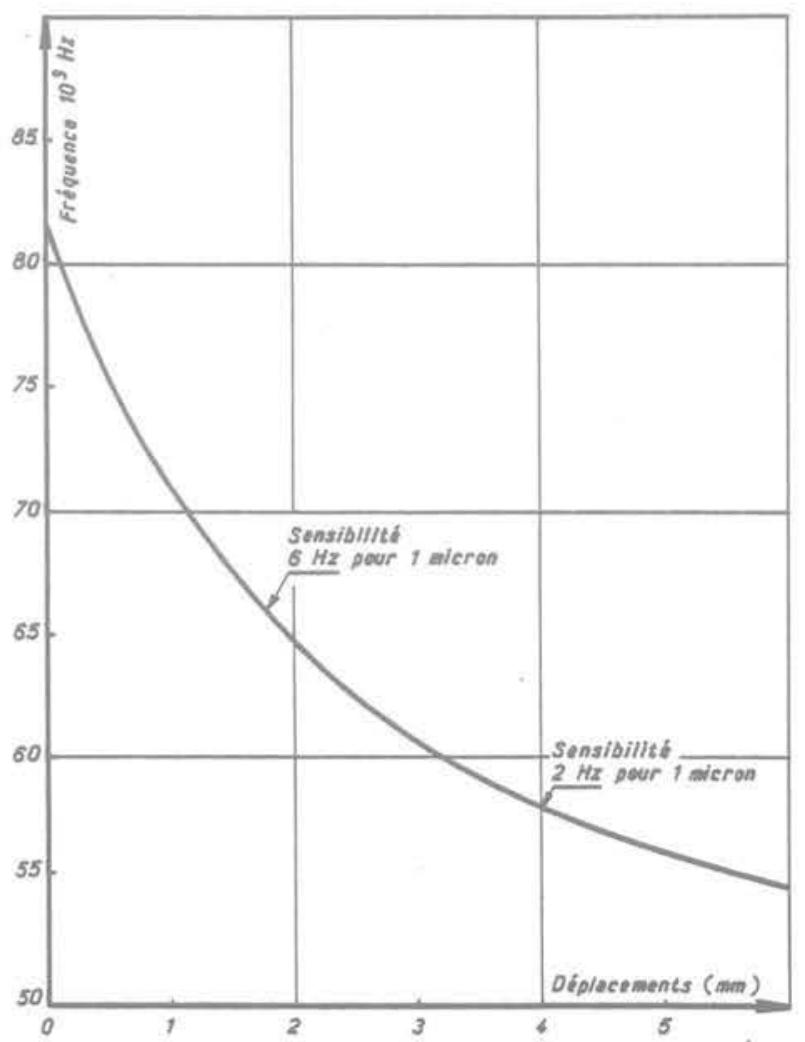

Fig. 5 Exemple de courbe de réponse du capteur 1 
Le processus consiste à gonfler la cellule à la pression préalablement déterminée pour être utilisée pendant le surcarottage, et à imposer un certain déplacement à l'aide de la vis micrométrique au capteur à étalonner. On fait ensuite varier ce déplacement par paliers, en mesurant les fréquences correspondantes, et ce jusqu'en fin de course du capteur ( $10 \mathrm{~mm}$ environ).

Cette méthode permet d'obtenir un étalonnage contrôlé ( $100 \mu$ chaque fois), sous une pression constante, et selon un processus proche de la mesure réelle.

La figure 5 donne un exemple de courbe d'étalonnage obtenue.

On note que la réponse de ce type de capteur n'est pas linéaire, et que la précision de mesure dépend de la plage de fonctionnement: pour les déplacements faibles, de un à deux millimètres, on a à peu près $6 \mathrm{~Hz}$ par micron, tandis que dans la plage de 4 à $5 \mathrm{~mm}$, on a seulement $2 \mathrm{~Hz}$ par micron.

Les conséquences de cette non-linéarité sont de deux ordres : en premier lieu, il convient d'essayer toujours de faire travailler la cellule dans la plage correspondant au début de course des capteurs, afin d'avoir la meilleure précision. Pour celà, il faut que la différence entre le diamètre extérieur de la cellule et le diamètre du trou soit minimale, c'est-à-dire que le diamètre du trou soit le plus possible de $97 \mathrm{~mm}$. D'autre part, un calculateur doit être utilisé pour convertir directement les fréquences mesurées en déplacements.

\section{Le matériel de mesure}

Le matériel nécessaire pour effectuer les mesures par la cellule CERCHAR peut se classer en deux groupes principaux :

- le premier concerne les travaux de forage; il comprend d'une part une sondeuse légère susceptible d'être dissociée en plusieurs éléments, équipée d'un moteur électrique puissant et alimentée par un groupe électrogène, et d'autre part un certain nombre de matériels de service, comme les carottiers, la pompe à eau, etc.;

- le deuxième est le système de saisie des données, piloté par un petit calculateur HP 9825. II permet d'abord la saisie automatique des mesures successivement sur tous les capteurs grâce à un scrutateur et un fréquencemètre, ensuite la transmission au jour et la conversion des Hertz en microns selon les courbes d'étalonnage déjà établies et stockées en mémoire, et enfin l'enregistrement des résultats sur cassettes pour les traiter ultérieurement et l'impression sous forme d'un listing des résultats des mesures. Afin que l'ingénieur au fond contrôle en permanence la validité des mesures pendant l'opération de surcarottage, l'information est simultanément visualisée au fond sur un écran de télévision.

Ce système informatique est transportable et normalement laissé au jour; mais il peut être éventuellement descendu dans les mines non-grisouteuses moyennant certaines précautions vis-à-vis des poussières et de I'humidité. La figure 6 représente un schéma d'ensemble du matériel utilisé.

Tableau 1

Quelques exemples des appareils des mesures des contraintes*

\begin{tabular}{l|l|l|l}
\hline \multicolumn{1}{c|}{ Appareils } & \multicolumn{1}{|c|}{$\begin{array}{c}\text { Type de mesures } \\
\text { effectuées }\end{array}$} & \multicolumn{1}{c}{$\begin{array}{c}\text { Principe de l'instrument } \\
\text { de mesure }\end{array}$} & \multicolumn{1}{c}{$\begin{array}{c}\text { Méthode de détermination } \\
\text { des contraintes }\end{array}$} \\
\hline $\begin{array}{l}\text { Déformètre Invar } \\
\text { D. C. O. T. }\end{array}$ & Déformation linéaire & Bobine d'induction & $\begin{array}{l}\text { Détente totale d'une surface } \\
\text { libre plane }\end{array}$ \\
\hline $\begin{array}{l}\text { Disque } \\
\text { photoélastique }\end{array}$ & Contraintes & Photoélasticité & $\begin{array}{l}\text { Détente totale d'une surface } \\
\text { libre plane ou bien du fond plat } \\
\text { d'un forage }\end{array}$ \\
\hline $\begin{array}{l}\text { Doorstopper } \\
\text { Talobre }\end{array}$ & Dilatations & $\begin{array}{l}\text { Résistances électriques } \\
\text { Jauges de déformation ohmiques }\end{array}$ & $\begin{array}{l}\text { Détente totale du fond plat } \\
\text { d'un forage }\end{array}$ \\
\hline $\begin{array}{l}\text { Vérin plat } \\
\text { linéaires }\end{array}$ & $\begin{array}{l}\text { Pression d'un liquide } \\
\text { et déformation linéaire }\end{array}$ & Manomètre et extensomètre & $\begin{array}{l}\text { Détente partielle par forage } \\
\text { d'une surface libre plane }\end{array}$ \\
\hline $\begin{array}{l}\text { Cellule "Universitétabsement des contraintes } \\
\text { du Minnesota" }\end{array}$ & $\begin{array}{l}\text { Déformations } \\
\text { diamétrales }\end{array}$ & $\begin{array}{l}\text { Lames encastrées avec jauges } \\
\text { de déformation ohmiques }\end{array}$ & $\begin{array}{l}\text { Détente totale d'un forage } \\
\text { libre }\end{array}$ \\
\hline $\begin{array}{l}\text { Cellule « Université } \\
\text { de Liègen }\end{array}$ & $\begin{array}{l}\text { Déformation } \\
\text { diamétrales } \\
\text { et déformation } \\
\text { longitudinale }\end{array}$ & $\begin{array}{l}\text { Lames encastrées avec jauges } \\
\text { de déformation ohmiques }\end{array}$ & $\begin{array}{l}\text { Détente totale d'un forage } \\
\text { libre }\end{array}$ \\
\hline
\end{tabular}

- D'après Bonnechère (2). 


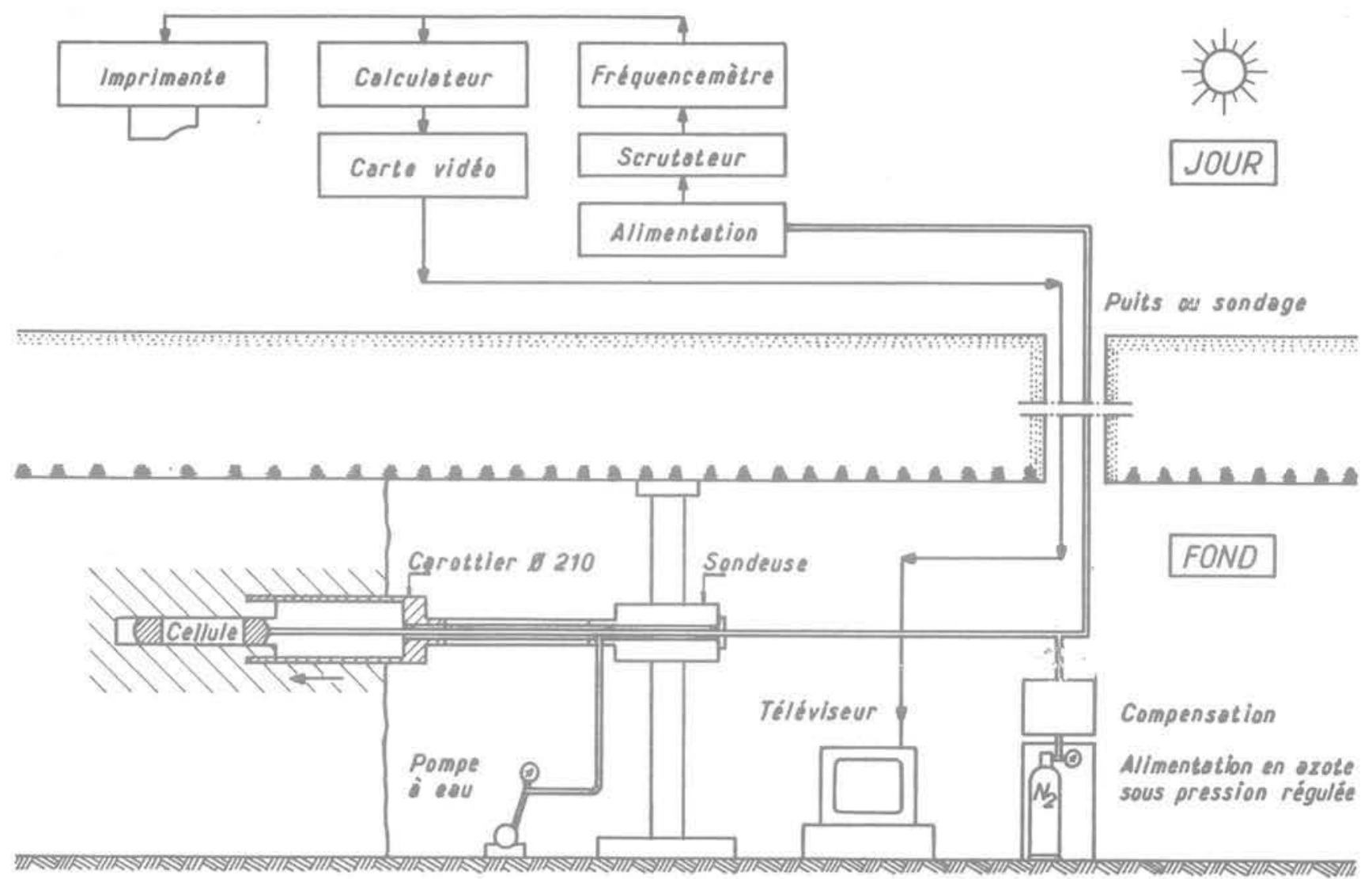

Fig. 6 Schéma d'ensemble du matériel de mesure de surcarottage

\section{Méthode d'interprétation des mesures}

Considérons un massif homogène, isotrope et linéairement élastique percé d'un trou circulaire de diamètre d et soumis à un état homogène de contraintes (figure 7).

La détente des contraintes appliquées induit une variation du diamètre du trou, qui est égale en module, mais de sens opposé, à la variation de diamètre qui avait été provoquée par l'application de ces contraintes sur le même massif initialement non-contraint.

Les relations entre la variation diamétrale $U$ et les contraintes appliquées $\sigma$ dans le repère local du trou, figure 7, s'écrivent Panek [4]; Himarastu et Ok [5] :

$U=F_{1} \cdot \sigma_{1}+F_{2} \cdot \sigma_{2}+F_{3} \cdot \sigma_{3}+F_{4} \cdot \tau_{13}$

avec

$F_{1}=\frac{d\left(1-v^{2}\right)}{E}(1+2 \cos 2 \theta)+\frac{d v^{2}}{E}$

$\mathrm{F}_{2}=-\frac{\mathrm{d} v}{\mathrm{E}}$

$F_{3}=\frac{d\left(1-v^{2}\right)}{E}(1-2 \cos 2 \theta)+\frac{d v^{2}}{E}$

$F_{4}=\frac{d\left(1-v^{2}\right)}{E}(4 \sin 2 \theta)$

où $\sigma_{1}, \sigma_{2}, \sigma_{3}, \tau_{13}$ sont définis sur la figure 7 .

$\mathrm{U}=$ variation diamétrale dans la direction $\theta$

$\mathrm{d}=$ diamètre du trou

$\mathrm{E}=$ module d'élasticité du matériau

$\nu=$ coefficient de Poisson du matériau.

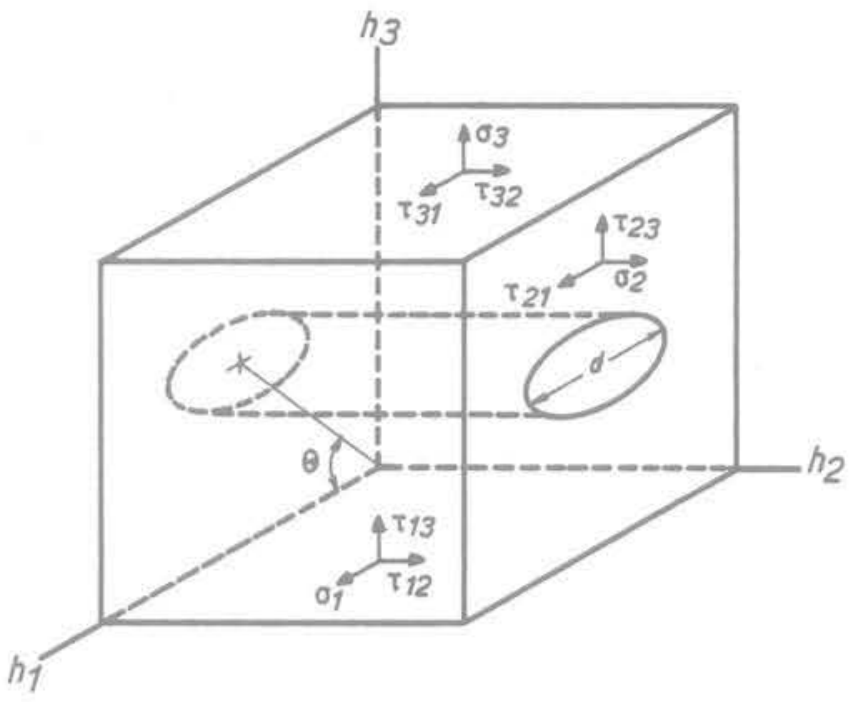

Fig. 7 Système de coordonnées de l'axe du trou

L'équation 1 est une relation paramétrique entre d'une part $U$ et d'autre part $\sigma_{1}, \sigma_{2}, \sigma_{3}$ et $\tau_{13}$. Cependant, elle ne permet pas de calculer ces quatre composantes sans une hypothèse supplémentaire, car seulement trois valeurs de $U$ sont indépendantes.

L'idée est donc d'effectuer des mesures dans d'autres trous et de rapporter toutes les mesures à un seul repère $(x, y, z)$, ce qui donne d'après les équations de transformation :

$U=J_{1} \sigma_{x}+J_{2} \sigma_{y}+J_{3} \sigma_{z}+J_{4} \tau_{x y}+J_{5} \tau_{y z}+J_{6} \tau_{z x}$ 
avec

$J_{1}=F_{1} \ell_{1}^{2}+F_{2} \ell_{2}^{2}+F_{3} \ell_{3}^{2}+F_{4} \ell_{1} \ell_{3}$

$J_{2}=F_{1} m_{1}^{2}+F_{2} m_{2}^{2}+F_{3} m_{3}^{2}+F_{4} m_{1} m_{3}$

$J_{3}=F_{1} n_{1}^{2}+F_{2} n_{2}^{2}+F_{3} n_{3}^{2}+F_{4} n_{1} n_{3}$

$J_{4}=2 F_{1} \ell_{1} m_{1}+2 F_{2} \ell_{2} m_{2}+2 F_{3} \ell_{3} m_{3}+F_{4}\left(\ell_{1} m_{3}+\ell_{3} m_{1}\right)$

$J_{5}=2 F_{1} m_{1} n_{1}+2 F_{2} m_{2} n_{2}+2 F_{3} m_{3} n_{3}+F_{4}\left(m_{1} n_{3}+m_{3} n_{1}\right)$

$J_{6}=2 F_{1} n_{1} \ell_{1}+2 F_{2} n_{2} \ell_{2}+2 F_{3} n_{3} \ell_{3}+F_{4}\left(n_{1} \ell_{3}+n_{3} \ell_{1}\right)$

oủ $\sigma_{x}, \sigma_{y}, \ldots, \tau_{z x}$ sont les composantes du tenseur des contraintes dans le repère $(x, y, z)$.

$\ell_{i}, m_{i}, n_{i}, i=1,2,3$ sont les cosinus directeurs de $h_{i}$ par rapport aux axes $x, y, z$ respectivement.

L'équation 2 exprime une liaison linéaire entre la variation diamétrale mesurée et les contraintes appliquées au massif. Chaque détermination de U correspond à certaines valeurs de $J_{i}$ qui dépendent seulement de la direction de mesure $\theta$ et les caractéristiques élastiques $\mathrm{E}$ et $v$. La détermination de $\mathrm{U}$ pour six conditions indépendantes permet d'obtenir par l'équation un système de six équations indépendantes. La résolution de ce système donnera les'six composantes du tenseur des contraintes.

La mesure des variations diamétrales dans un trou par la cellule CERCHAR (3 directions différentes) ne fournit que trois conditions indépendantes. Dans ces conditions, la détermination de ces six équations indépendantes peut être obtenue par deux trous au minimum, à condition qu'ils ne soient ni parallèles ni perpendiculaires.

Le problème de la validité des mesures et la précision du calcul des contraintes, c'est-à-dire la représentativité de la valeur calculée par rapport à la valeur réelle peut être abordé de deux manières. L'une consiste à améliorer la précision en mesurant $U$ dans plusieurs trous et en calculant à l'aide de la méthode des moindres carrés une valeur moyenne pour chaque composante du tenseur des contraintes. L'application de la méthode des moindres carrés permet d'évaluer la dispersion des composantes calculées et de déterminer leur intervalle de confiance. D'autres analyses statistiques sont également possibles avec ce type de calcul. L'autre approche traite le problème par la simplification du calcul en faisant intervenir des hypothèses supplémentaires. Quant à la précision des mesures, elle devient alors une fonction de la validité de la simplification par rapport aux conditions in situ en particulier la géométrie de l'ouvrage.

Un programme informatique a été décrit pour exécuter les procédures de ce calcul. II présente la possibilité d'effectuer les calculs avec des nombres de mesures variables et selon différentes hypothèses de répartition des contraintes.

Pour déterminer les contraintes in situ à l'aide du calcul précédent, il faut compléter la mesure des déformations diamétrales par la connaissance des paramètres élastiques du matériau $(E, v)$. La détermination de ces paramètres représente un des problèmes fondamentaux de la mesure des contraintes in situ. La précision de la détermination des contraintes est directement liée à la précision avec laquelle ces paramètres sont déterminés. Si toutefois, la variation du coefficient de Poisson $v$ est relativement limitée, la valeur du module d'élasticité varie beaucoup; elle dépend de la structure des roches et peut-être également de l'état des contraintes in situ (M. Borecki et al).

\section{Détermination des paramètres élastiques du matériau $(\mathrm{E}, v)$}

II existe de nombreux moyens de détermination des caractéristiques élastiques des roches, au laboratoire ou in situ, qui tiennent compte de la spécificité du problème. La plupart des méthodes de laboratoire sont basées sur l'utilisation de carottes issues du forage lui-même, en simulant le champs des contraintes in situ, soit dans un essai monoaxial (Fitzpatrick [6]), soit dans un essai triaxial (Obert [7]). L'inconvénient principal des mesures de laboratoires réside dans la difficulté de simuler exactement les conditions in situ (en particulier, la température, I'humidité et peut-être la répartition des contraintes), tandis que la mesure en place prend en compte les conditions locales réelles, en particulier la fissuration, l'effet d'échelle et l'anisotropie du matériau. Plusieurs méthodes permettant de déterminer le module in situ sont proposées dans la bibliographie, comme par exemple, le vérin plat (Bonvallet [3]), l'essai à la plaque (Van Heerden [8]) et l'essai dilatométrique en trou de sondage (Duffaut [9]). II faut toutefois noter que toutes ces méthodes nécessitent l'emploi d'un certain nombre de matériels spécialisés dans des conditions parfois difficiles.

La cellule CERCHAR a l'avantage de fonctionner comme un dilatomètre; cette possibilité peut être utilisée pour mesurer le module pressiométrique de la roche dans le trou même, avant de commencer l'opération du surcarottage proprement dite. Après l'installation de la cellule dans le forage de petit diamètre et la détermination des conditions initiales, un essai cyclique de pressiométrie (charge-décharge) peut être effectué. De cet essai, on déduit une pente de la courbe pressiométrique que l'on utilise dans la formule 3 développée à partir du modèle du cylindre épais :

$E=(1+v) d \cdot \frac{\Delta P}{\Delta U}$

où

$E=$ module d'élasticité

$\mathrm{d}=$ diamètre du trou

$\mathrm{P}=$ pression appliquée

$\mathrm{U}=$ variation diamétrale mesurée

$\nu=$ coefficient de Poisson

En ce qui concerne le coefficient de Poisson $v$, il est déterminé en laboratoire sur éprouvettes. Afin d'améliorer la précision des résultats, plusieurs essais sont effectués.

\section{Essais en laboratoire}

Un certain nombre d'essais ont été réalisés en laboratoire sous presse. L'objectif était d'étudier le fonctionnement de la cellule et de contrôler la fiabilité de l'ensemble de l'appareillage utilisé avec cette cellule, sous un état de contrainte connu (monoaxial) avant son emploi in situ.

Pour atteindre cet objectif, 3 matériaux de caractéristiques différentes ont été choisis. Cinq blocs prismatiques de dimensions $40 \times 40 \times 60 \mathrm{~cm}$ percés d'un trou de diamètre $97 \mathrm{~mm}$ au milieu, ont été découpés. Au préalable, des essais de caractérisation ont été effectués sur des éprouvettes représentatives des 3 matériaux, en vue de connaître leurs caractéristiques et de déterminer la charge maximale à appliquer sur chaque bloc pour rester dans le domaine élastique. 


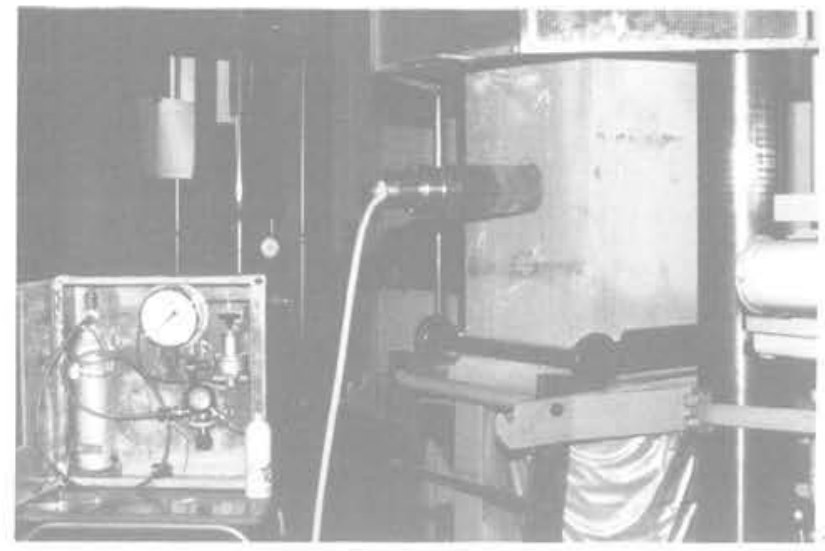

Fig. 8 La cellule dans le bloc de grès sous la presse

Déterminer la variation de diamètre intervenant dans le trou lorsque l'on supprime les contraintes appliquées au bloc revient à déterminer la variation diamétrale provoquée par le surcarottage.

L'opération de surcarottage a donc été simulée en portant la charge initiale à la valeur maximale possible, et en mesurant les variations de diamètre pendant la décharge. La figure 8 montre une vue de la cellule dans un bloc de grès de Niderviller sous la presse. Un exemple de la réponse de la cellule dans un bloc de béton est illustré par la figure 9 .

L'examen des résultats obtenus montre que la variation diamétrale mesurée par la cellule est une fonction linéaire de la charge appliquée quel que soit le matériau, même pour des faibles niveaux de contraintes. Mais la pente de la courbe obtenue dépend du module d'élasticité de ce matériau. Nous avons constaté que la pente de la courbe correspondait à un module supérieur à celui mesuré sur éprouvettes en laboratoîre.

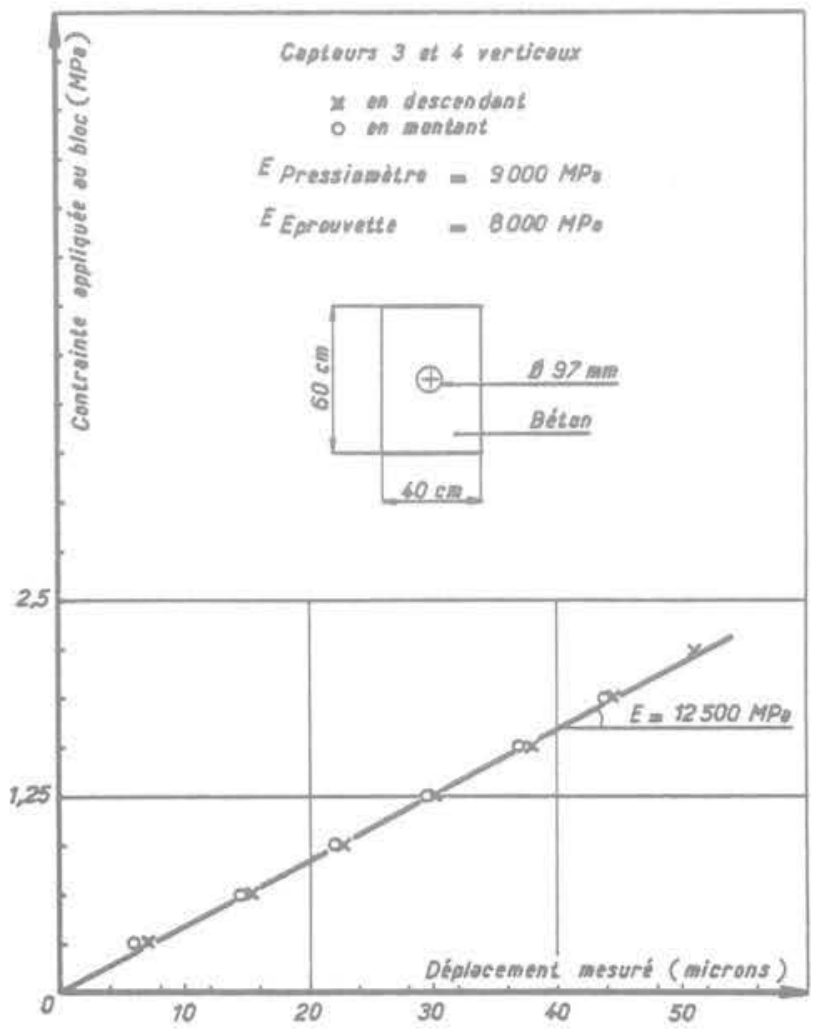

Fig. 9 Exemple de courbe de réponse de la cellule dans le béton
Dans la plupart des cas, il est impossible de déterminer un module pressiométrique dans le bloc sous la presse en raison de la fissuration que provoque l'essai, et qui se traduit par un éclatement du bloc.

\section{Mesure in situ}

L'ensemble de l'appareillage a été utilisé pour mesurer les contraintes in situ. Quelques campagnes de mesure ont été réalisées dans les carrières souterraines à faible profondeur et dans les mines à profondeur moyenne, où plusieurs séries de mesures de pressiométrie et de surcarottage ont été effectuées dans les piliers (tableau II ci-après). Parmi ces mesures, quelquesunes ont été réalisées en des points pour lesquels des mesures par la méthode du vérin plat avaient déjà été effectuées. La figure 10 illustre un exemple de courbe de pressiométrie obtenue dans la craie oủ la pression maximale a été limitée à $1,1 \mathrm{MPa}$. On remarque que le module déterminé par la première montée est très faible, ce quî peut être dû au tassement du matériau.

Tableau II

Caractéristiques élémentaires des sites étudiés

\begin{tabular}{l|c|c}
\hline \multicolumn{1}{c|}{ Cas étudié } & $\begin{array}{c}\text { Taux de } \\
\text { défruitement (\%) }\end{array}$ & $\begin{array}{c}\text { Profondeur } \\
\text { en mètres }\end{array}$ \\
\hline $\begin{array}{l}\text { Lezennes (59) } \\
\text { (craie blanche) }\end{array}$ & 81 & 11,5 \\
\hline $\begin{array}{l}\text { Extreux (59) } \\
\text { (craie grise) }\end{array}$ & 80 & 19,8 \\
\hline $\begin{array}{l}\text { Vaillon (60) } \\
\text { (calcaire) }\end{array}$ & 68 & 7 \\
\hline $\begin{array}{l}\text { Grozon (39) } \\
\text { (gypse) }\end{array}$ & $63-75$ & 55 \\
\hline $\begin{array}{l}\text { Varangéville (54) } \\
\text { sel }\end{array}$ & 75 & 192 \\
\hline
\end{tabular}

Le module pressiométrique est calculé en prenant la moyenne des $2^{\circ}$ et $3^{\circ}$ descentes entre 0,5 et $1,1 \mathrm{MPa}$. On note également que malgré le faible niveau de la pression appliquée, le module déterminé est en bonne concordance avec celui déterminé par la méthode du vérin plat au même endroit.

Quelques exemples des courbes caractéristiques du surcarottage dans différents états de contraintes sont donnés sur les figures 11,12 et 13 . Ces courbes représentent les déplacements diamétraux mesurés par la cellule, en fonction de l'avancement du carottier. Trois mesures par centimètre d'avancement ont été enregistrées, ce qui représente plus d'une centaine de points pour établir chaque courbe complète. L'analyse de ces courbes montre que la réponse de la cellule en fonction du milieu rocheux est en parfait accord avec la courbe théorique du surcarottage obtenue à partir d'un modèle par éléments finis (Blackwood [10]). 


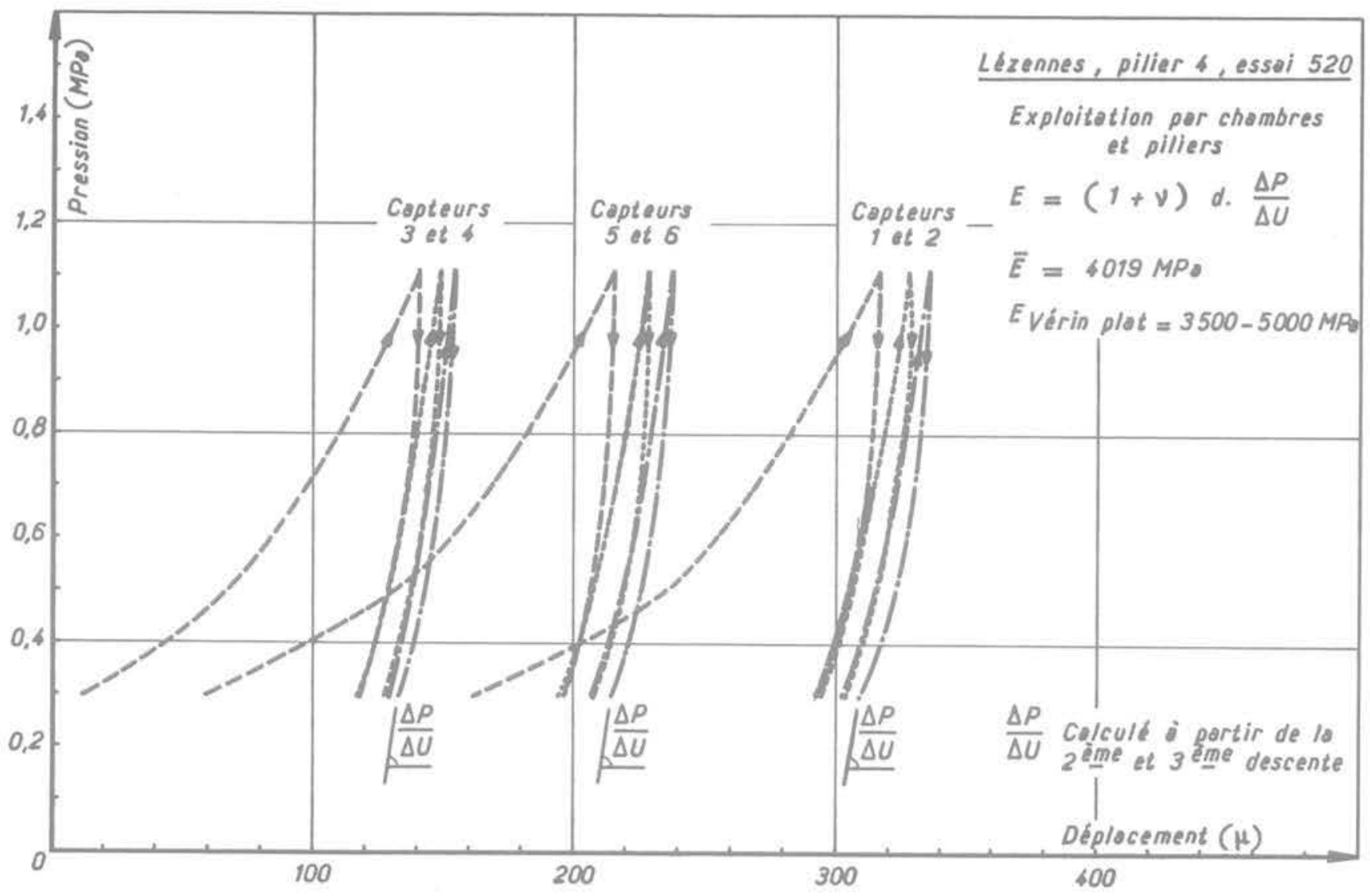

Fig. 10 Exemple de courbes pressiométriques obtenues dans la craie de Lézennes

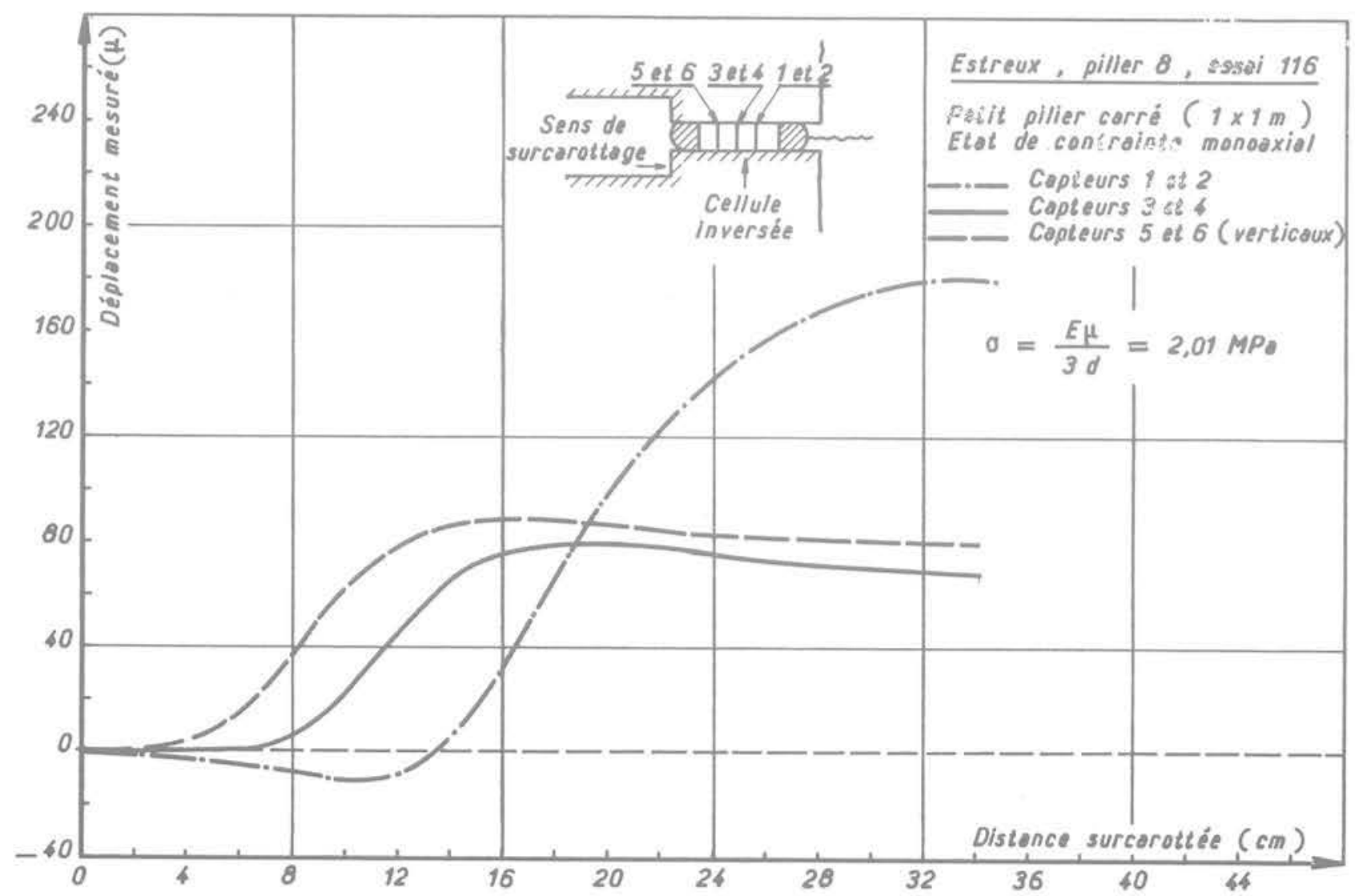

Fig. 11 Courbes caractéristiques du surcarottage dans la craie d'Estreux 


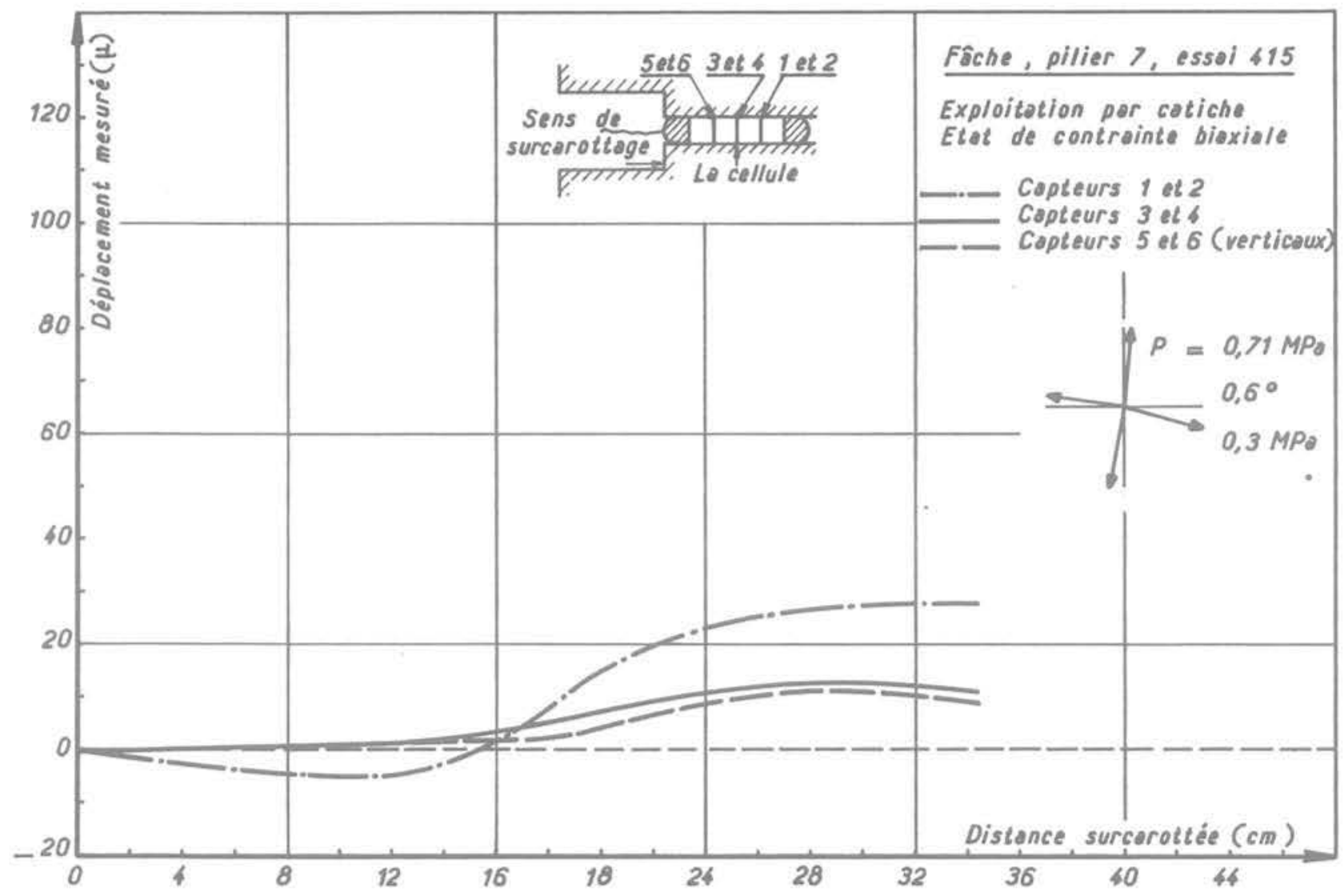

Fig. 12 Courbes caractéristiques du surcarottage dans la craie de Fache

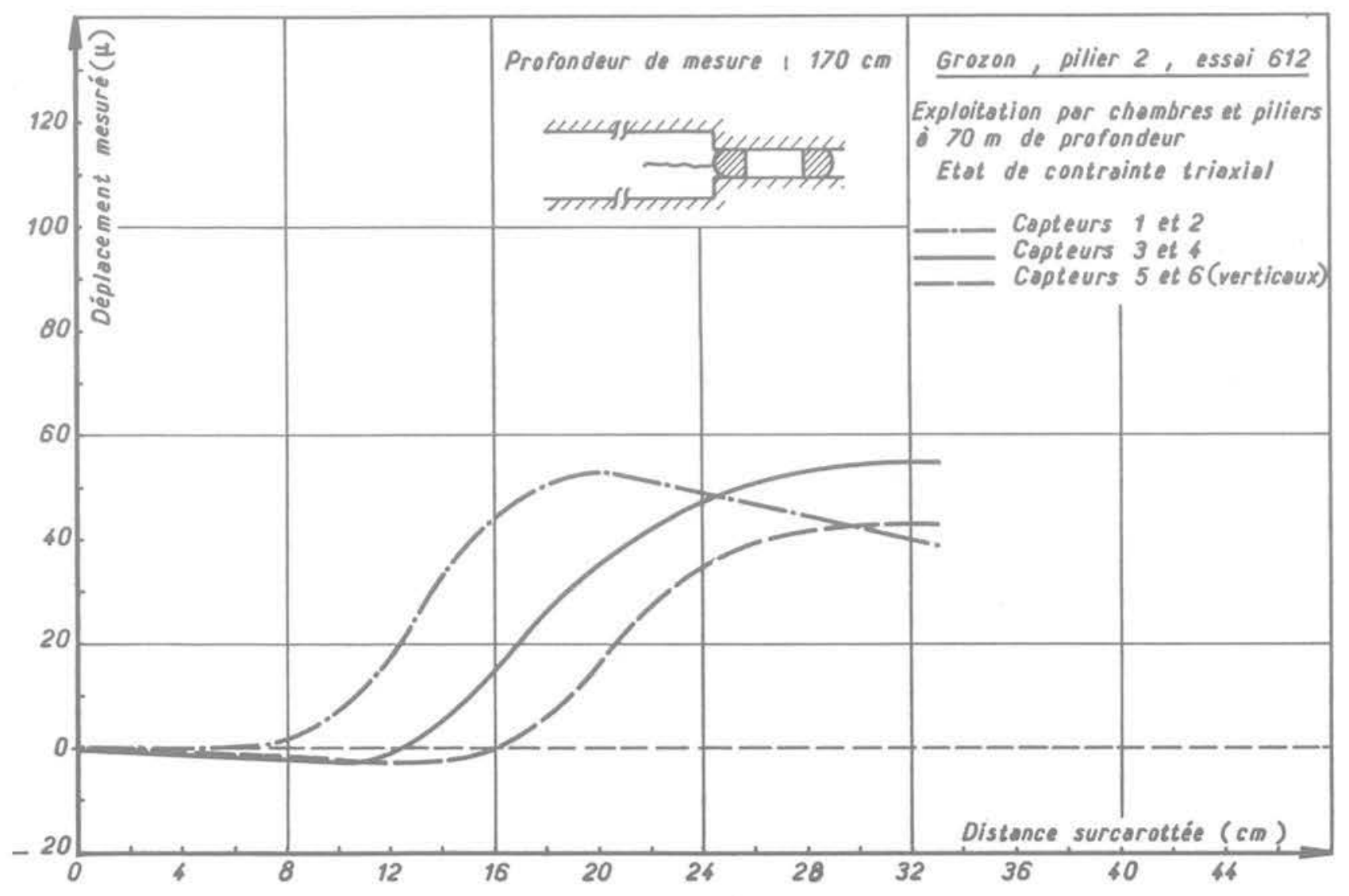

Fig. 13 Courbes caractéristiques du surcarottage dans le gypse de Grozon 


\section{Conclusion}

La cellule CERCHAR et l'ensemble de l'appareillage de mesure présentent une originalité qui satisfait toutes les exigences d'une méthode de mesure in situ, c'està-dire :

- une mesure continue est assurée par l'unité informatique, ce qui permet d'établir la courbe de surcarottage avec plus d'une centaine de points;

- les résultats sont simultanément affichés au chantier sur un téléviseur ce qui assure un contrôle de l'opération;

- la cellule présente une bonne précision sous très faible pression:

- la double utilisation de la cellule en tant que cellule de mesure radiale et comme dilatomètre permet la détermination du module d'élasticité sans aucun matériel supplémentaire:

- aucun collage n'est utilisé pour fixer la cellule. Le contact entre les capteurs et la paroi du trou est assuré par la pression de l'azote comprimé;

- les dérives possibles des capteurs de mesure pendant l'opération du surcarottage peuvent être corrigées grâce au capteur de référence;

- l'étalonnage des capteurs de mesure est très simple et demande un minimum de matériel.

Enfin, pour contrôler l'hypothèse de l'élasticité linéaire du matériau, il est nécessaire de compléter la procédure de mesure par la remise en pression de l'anneau de terrạin surcarotté à l'aide de vérins courbes. Un dispositif (représenté sur la fig. 14) a été mis au point qui permet effectivement de contrôler in situ la linéarité du comportement du matériau, et d'effectuer les mesures en utilisant seulement l'hypothèse de la réversibilité des déformations.

\section{Références bibliographiques}

[1] LEEMAN - « The measurement of stress in rock $"$ J.S. Afr. Instr. Min and Met.

Part 1. The principles of rock stress measurements. Sept. 1964.

Part 2. Borehole rock stress measuring instruments. Sept. 1964.

Part 3. The results of some rock stress investigation. Nov. 1964.

[2] BONNECHERE - *Contribution à la détermination de l'état de contrainte des massifs rocheux. » Thèse en Science devant I'Univ. de Liège 1971.

[3] BONVALLET - * Critères de stabilité des exploitations souterraines à faible profondeur - Application au cas des carrières souterraines du Nord. "

Thèse de Doct. Ing. I. N.P. L. 1978.

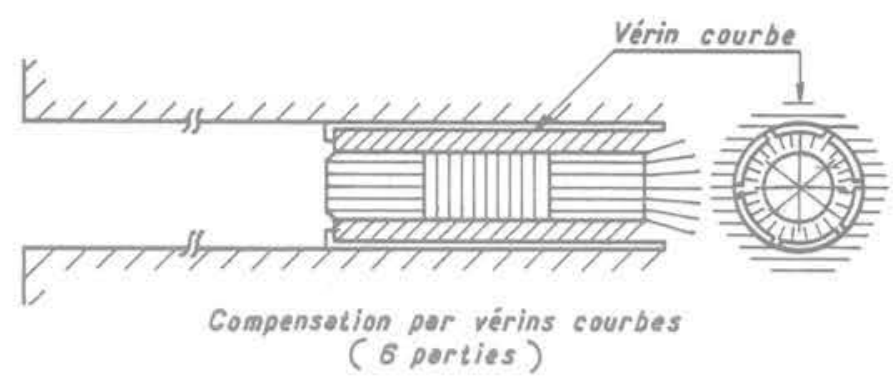

Fig. 14 Remise en pression de l'anneau de terrain surcarotté par des vérins courbes

[4] PANEK L, A. - " Calculation of the average ground stress components from measurements of the diametral deformation of a drill hole. $n$

Rep, of Inv. 6732. U.S. Bur. Mines, 41 pp., 1966.

[5] HIRAMATSU and OKA - "Determination of the stress in rock unaffected by borehales or drifts, from measured strains or deformations. $n$ Int. J. Rock Mech. Min. Sci. Vol. 5 , pp. $337,1968$.

[6] FITZPATRICK - " Biaxial Device for determining the modulus of elasticity of stress relief cores.

U.S. BU. Mines Rept of Inv. 6128, 13 pp. 1962.

[7] OBERT L. - " Triaxial method for determing the elastic constants of stress relief cores. "

U.S. Bu. Mines Rep. of Inv. 6490, 22 pp., 1964.

[8] VAN HEERDEN W. L., MASCHER R. K. A. - - In situ modulus determination, a case history. Elandsberg schema, south africa. »

Proceedings of Int. Cong. on Rock Mechanics; Montreux 1979, vol. 2, pp. 705-710.

[9] DUFFAUT P., COMES G. - " Comparaison de la déformabilité statique d'un matériau de fondation mesurée en place sur le parement d'une excavation et à la paroi d'un sondage.

Comptes rendus, for Cong. Sco. Int. Meca Roche, Lisbonne, 1966, vol. 1, pp. 399-404.

[10] BLACKWOOD R.L. - « Diagnostic stress relief curves in stress measurement by overcoring. $x$ Int. J. Rock. Mech. Min., Sci. vol. 15, pp. 205-209, 1978.

[11] MARCIN BORECKI and ANTONI KIDYBINSKI "Problems of stress measurements in rocks taken in Polish Coal Mining Industry, "

Comptes rendus, $1^{\text {er }}$ Cong. Soc. Int. Meca Roches, Lisbonne, vol. 11, p. 9-16, 1966. 